\title{
The Star Formation History in Nearby Dwarf Galaxies: the Fossil Record in the Color-Magnitude Diagram
}

\author{
A. Aparicio \\ Instituto de Astrofísica de Canarias, E38200 La Laguna, Tenerife, \\ Canary Islands, Spain
}

\begin{abstract}
.
Dwarf galaxies may play a key role in the formation and evolution of bigger systems. This makes knowing how they form and evolve and, in particular, how their star formation histories (SFHs) have proceeded since their birth, a topic of major interest. For nearby galaxies, the colormagnitude diagram (CMD) contains stars formed over all their lifetime. It is hence a fossil record of their SFHs. The analysis with synthetic CMDs provides a powerful tool to retrieve them.

In this paper, I discuss the critical issues related to the computation of synthetic CMDs, present a short summary of the currently available results for the SFH extending the full life of galaxies and make a few critical considerations about the powerfulness and draw-backs of the method.
\end{abstract}

\section{Introduction}

At the low end of the luminosity function, dwarf galaxies seem to be by far the most numerous in the Universe. At least, this is the case in the Local Group, where $90 \%$ of its about 40 members are dwarfs. Besides this, the hierarchic scenarios of galaxy formation, such as an Universe dominated by cold dark matter, predict that dwarf galaxies are likely to be the first structures to form, arising from $1 \sigma$ fluctuations in the density distribution of the primeval Universe and that they would proceed afterwards to form larger galaxies. These two reasons are enough to pay a preferential attention to these objects. The fortunate fact that the Local Group contains a wide variety of dwarf galaxies provides a good opportunity to study them in depth and to test the predictions provided by Cosmology about the formation and evolution of galaxies.

Containing stars born over all the life-time of a galaxy, the color-magnitude diagram (CMD) is a fossil record of the the star formation history ( $\mathrm{SFH}$ ), which is in turn closely related with the evolution and, in the last instance, with the galaxy formation process itself. The best tool for deciphering that record is the analysis with synthetic CMDs, which can simulate any arbitrary input SFH (Aparicio et al. 1996; Gallart et al. 1999b). The fact that this work can only be performed for the most nearby galaxies, makes still more relevant the study of the Local Group and its close neighborhood.

In this paper, I will review the synthetic CMD method, its virtues and its shortcomings (Sec. 2 and 3); give a summary of results obtained for galaxies 

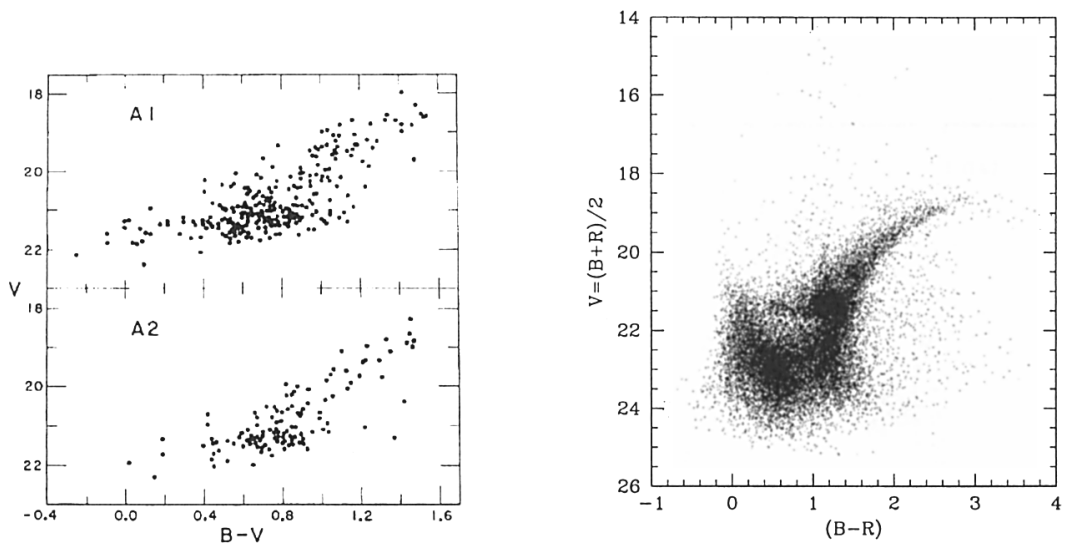

Figure 1. CMDs of the Fornax dwarf galaxy showing the evolution in the data quality in the last decade. Left panel is from Buonanno et al. (1985); right panel is from Stetson et al. (1998). The latter shows the present day state of the art for wide-field ground-based observations.

for which the SFH extending their whole life is available through the synthetic CMDs technique (Sec. 4); make a concise statement about future work (Sec. 5) and a short critical discussion of some of the preconceptions and misconceptions related to the SFH of galaxies and its determination (Sec. 6).

\section{Star Formation History and Color-Magnitude Diagram}

The recent growing interest in obtaining detailed SFHs of nearby galaxies has been, at least partially, due to the fast development of our capabilities to determine them. This is linked to the improvement of both our observational resources and our theoretical tools:

- From the observational side, the better image quality, instrument sensitivity and field of view now available, compared with those of a decade ago, has produced a great increase in the depth and accuracy of CMDs. To illustrate this, Figs. 1 and 2 show the CMDs of two nearby dwarfs: Fornax and Leo I. In both cases, the left-side panels show some decade-old data and the right-side panels, the latest available data. Not only the new data provide information for the main sequence (MS) reaching the oldest stars, but also a good sampling of fast stellar evolutionary phases, such as the red-clump (RC) and blue-loop (BL) of core He-burning intermediate-age stars, or the asymptotic giant branch (AGB).

- From the theoretical side, the improvement concerns two main issues: (i) the availability of stellar evolution libraries with a wide coverage in age and metallicity, and including the calculation of advanced phases such as the horizontal-branch (HB) or the AGB and (ii) the development of codes 

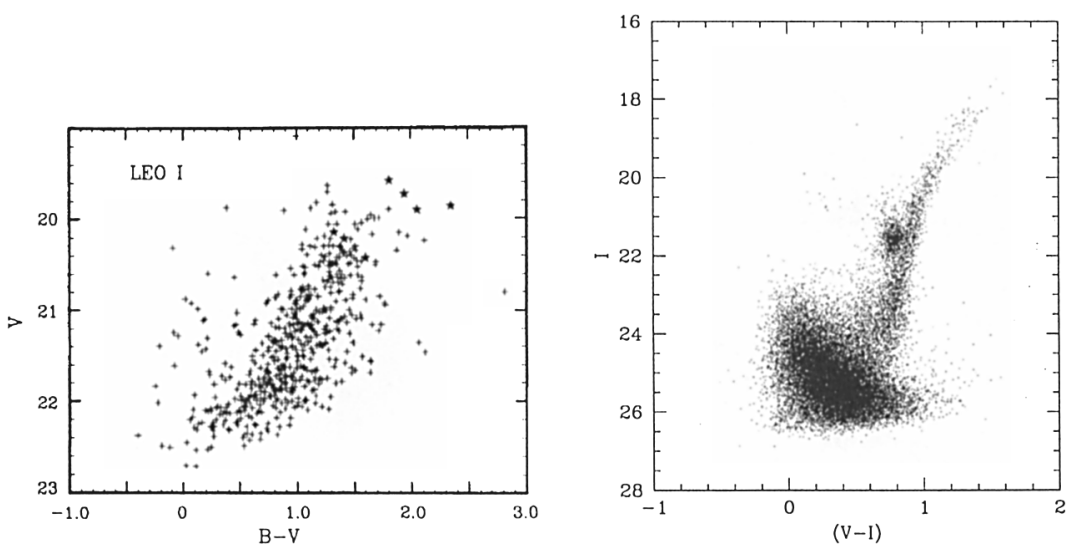

Figure 2. CMDs of Leo I dwarf galaxy showing the evolution in the data quality in the last decade. Left panel is from Fox \& Pritchet (1987); right panel is from Gallart et al. (1999a). The latter shows the present day state of the art for HST observations.

for computation of synthetic CMDs. Details on the analysis with synthetic CMDs are given in Sec. 3 .

The information on the SFH contained in CMDs and the potential of the synthetic CMDs technique is visualized in Fig. 3. It shows the stellar population of a simulated galaxy in which the star formation had proceeded at a constant rate from 15 Gyr ago to date and in which the metallicity had increased linearly from $Z=0.0001$ to $Z=0.004$. In each panel, stars with ages in the given intervals (in Gyr) are plotted. The changes not only in the MS extension, but also in the shape of other features like the $\mathrm{RC}$, the $\mathrm{HB}$, the $\mathrm{BL}$, the red giant branch (RGB) or the AGB are clear. It is the analysis of the distribution of the stars across the CMD, which is related to these changes, that can be used to derive the SFH. For details about the features of the CMD and the age distribution of stars populating it see Chiosi et al. (1992); Aparicio \& Gallart (1994); Aparicio et al. (1996); Da Costa (1998) and Mateo (1998).

\section{Synthetic CMDs: the Method}

The method to derive the SFH from synthetic CMDs is composed of three main steps: (i) computation of the synthetic CMD itself, reproducing a given $\mathrm{SFH}$ and using stellar evolutionary models; (ii) simulation of observational effects into the synthetic CMD and (iii) comparison of the observational and the synthetic CMD to determine the acceptable and/or best models. Careful handling of the problems arising in each of these steps determines the success of the final solution. I will briefly discuss these problems in the present section. 


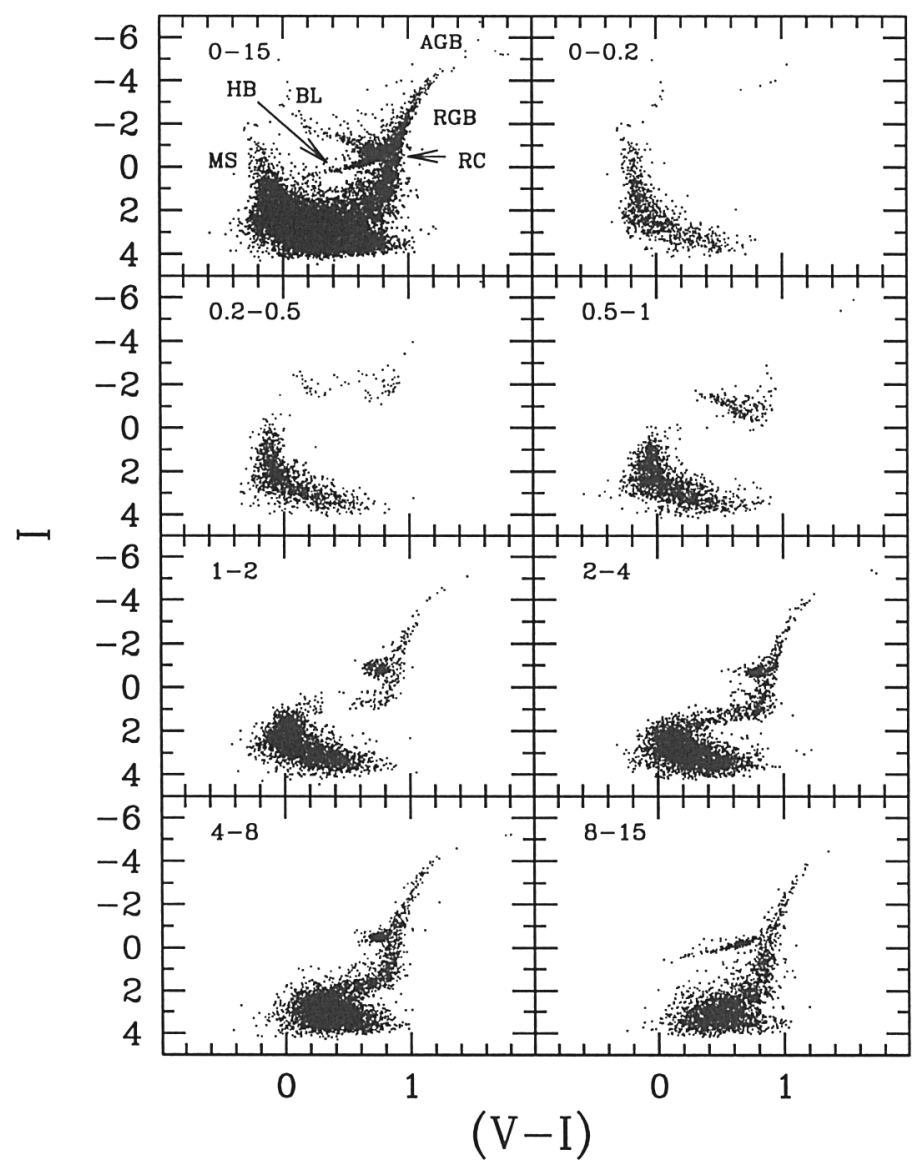

Figure 3. Model CMDs for a constant SFR from 15 Gyr ago to the present time and a linearly increasing $Z(t)$ from $Z=0.0001$ to $Z=$ 0.004. In each panel stars with ages in the given interval (in Gyr) are plotted. Observational effects have been simulated as described in Sec. 3.2 . 


\subsection{Computing the Synthetic CMD}

The main input for the synthetic CMD computation is the SFH, which can be understood as a combination of several simpler functions: the SFR $\psi(t)$; the chemical enrichment law $Z(t)$; and the initial mass function (IMF) $\phi(m)$. Together with them, a function $\beta(f, q)$, controlling the fraction and mass ratio distribution of binary stars is also relevant. In the standard procedure, a montecarlo generator is then used to produce, for every star $i$, random values of the mass $m_{i}$ and the time of birth $t_{i}$, according to $\phi(m)$ and $\psi(t)$, respectively. Then, $t_{i}$ is used to determine the metallicity $Z_{i}$ through $Z(t)$. Later on, the binary nature of the star and, eventually, the mass of its companion, is determined using also a monte-carlo generator and $\beta(f, q)$.

At this point, the stellar evolution models are used first to determine whether the star is now alive. If so, an interpolation in the age-mass-metallicity evolution grid space is performed to determine first, the stellar evolution phase and then, the luminosity and effective temperature of the star. Finally, bolometric corrections are applied to obtain the magnitudes and color indices.

The critical point in this procedure is the interpolation inside a complete grid of stellar evolution models. To achieve a good representation of the observed data, such interpolation must be done both in age and metallicity to produce a smooth CMD containing the information of stars of any age and Z, and to be able to test any desired chemical enrichment law. Otherwise, a lumpy synthetic CMD would result. It would not give a good matching to the observational data and hence a reliable solution for the SFH could not be obtained. Besides this, it is not superfluous to note that the stellar evolutionary models must include accurate calculation of the shorter living phases, that can provide useful insight on the SFH. The RC, BL and AGB are typical examples of such phases.

The BL and the AGB deserve some comment. Both are quite sensitive to age and metallicity and notably contribute to the age-metallicity resolution when the lower MS is not accessible. However, colors of the BL stars depend on metallicity through parameters difficult to control. As a result, their color distribution can not be used as a confident indicator of the absolute metallicity distribution, although their luminosity distribution and their total number are good indicators of $\psi(t)$ for stars younger than $\sim 1$ Gyr. Similarly, colors and life-times of AGBs depend on the mass loss, again difficult to parameterize, and on the bolometric corrections, ill-known for the very red stars. Nevertheless, their number and relative distribution provide useful constraints on $\psi(t)$ for intermediate to old ages. In summary, the BL and the AGB provide good insight on the SFH, but uncertainties in the way they are distributed in the CMD imply that only rough estimates can be obtained.

\subsection{Observational Effects}

Martínez-Delgado \& Aparicio (1997) show how dramatic the consequences can be of neglecting observational effects in the analysis of a stellar population with CMDs. Including all the sources of uncertainty affecting the photometry of stars, observational effects must be simulated into the synthetic CMDs before comparison with the observations. While some of these effects are produced at the detector level (random noise, flat-field, bad pixels) or are simple results of statistics (poissonian signal-to-noise ratio), other, like crowding, are intrinsic 
to the observed object (surface density, luminosity, and color distributions of stars) or depend on specific observational conditions (diffraction limit for spacebased telescopes or the atmospheric seeing for ground-based telescopes). In fact, crowding is the effect usually imposing the ultimate limit to the photometry.

Observational effects are mainly of three kinds:

- Loss of stars, which mainly affects faint objects. The fraction of measured to existing stars as a function of magnitude is the completeness factor $\Lambda$.

- Magnitude and color shifts $(\delta)$, which depend on the magnitude and color of the stars. In general, stars are measured brighter than they are; blue stars are measured redder and red stars are measured bluer.

- Total (external) errors $(\Sigma)$ of the photometry, which are larger than the internal errors $(\sigma)$ and which are not related to them in a simple way.

The 'artificial stars' procedure used to characterize these effects (Stetson 1994), and their trends and the way to introduce them in the synthetic CMDs (Aparicio \& Gallart 1995; Gallart et al. 1996a), have been extensively written about. Suffice to note here that $\Lambda, \Sigma$ and $\delta$ are related to the distribution of color and magnitudes of the stellar population and with crowding, hence with the stellar surface density and instrumental resolution. The adequate simulation of observational effects requires the information of a large number of artificial stars, because $\delta$ and $\Sigma$ follow bidimensional distributions that vary across the CMD. Figures 7 and 8 of Aparicio \& Gallart (1995) show the trends of the observational effects on CMDs of artificial stars.

Provided that a large number of trials (typically several tens of thousands of artificial stars) has been done, we have proposed a simple, non-parametric method to simulate the observational effects in the synthetic CMDs. It is described in detail in Aparicio \& Gallart (1995) and in Gallart et al. (1996a). In short, the method consists of applying, to each star in the synthetic CMD, the shifts in color and magnitude observed in an artificial star picked up randomly from a subsample of artificial stars with similar color and magnitude. The powerfulness of the method resides in the empirical approach, with no modeling or analytical approximations introduced in the simulation process. It provides a full representation of all the observational effects in the synthetic CMD.

A refinement of the method consists of using the synthetic CMD itself as the list of artificial stars to be injected in the real images. The advantage of this approach is that it is free of sampling distortions that would appear in the most densely populated regions of the CMD (see Martínez-Delgado et al. 1999).

\subsection{Solving the SFH: Comparison of Synthetic and Observational CMDs}

The final step is searching for the SFH which produces the model CMD that best matches the observational one. Usually, the solution is not unique, as a result of the many degeneracies intrinsic to the CMDs themselves and uncertainties in the stellar evolution models. A strong limitation comes from the age-metallicity degeneracy in the RGB and can only be solved in a fully satisfactory way if accurate photometry of the MS is available down to the oldest turn-offs. But 
besides these unavoidable intrinsic limitations, the method followed to determine which are the acceptable synthetic CMDs and/or best reproducing the data introduces a new source of uncertainty.

Several of the methods used to compare model and observational CMDs treat or consider the diagrams as bidimensional (or n-dimensional in the general case if several color indices are used) distributions of points with Gaussian errors and use different techniques to search for good models. Tolstoy \& Saha (1996) use Bayesian inference to calculate the likelihood of model CMDs to be good representations of the data (see also Tolstoy et al. 1998 for a recent application); Serra-Ricart et al. (1996) apply a neural network technique to minimize a $\chi^{2}$; $\mathrm{Ng}$ (1998) propose a $\chi^{2}$ combined with a Poisson merit function to search for the best model and Hernández et al. (1999a) apply a variational method to maximize the likelihood.

The advantage of these approaches is that they are general methods requiring no human intervention for the comparison process: just two distributions of data points are compared with no more assumptions. The draw-back, in my opinion, is that, as a matter of fact, not all the data points can be considered on an equal basis, because different stellar evolutionary phases are known with different accuracy. For example, the color distribution and blue extension of the $\mathrm{BL}$ of intermediate mass and massive core He-burning stars, is quite sensitive to metallicity and to not well-known details in the stellar evolution models. But the lifetime of the stars in this phase, which is a function of mass and hence of age, is known with good confidence. If a method just comparing distributions of points is used, a big weight is given to the badly known details of the stellar evolution models and, consequently, the resulting SFH would be affected by this. But if, due to the uncertainties, these stellar evolutionary phases are removed from the comparison, the information provided by the distribution of these stars in luminosity is lost (see the discussion by $\mathrm{Ng} 1998$ ).

It is for these reasons why we usually prefer methods of comparison that allow human intervention to decide how different groups of stars have to be considered. The draw-back is that results could contain some subjective effects. The advantage is that suitable weights can be given to each evolutionary phase. In our approach, a good fit of the position and shape of the main features in the CMD is also used to impose a first limit to the range of possible solutions for the $\mathrm{SFH}$. Then several regions are defined in the CMDs and the numbers of stars in them are compared through a $\chi^{2}$ or a least squares method. The key point of the approach is that the size and location of the bins is decided considering the information provided by the different stellar evolutionary phases. While a fine grain binning is done in the MS area, larger boxes are used for the upper AGBs and a set of bins in luminosity but with no resolution in color is employed for the BL. A further advantage is that many age intervals can be studied through stars in different stellar evolutionary phases. In those cases, the solutions can be compared as a test of consistency. Examples of studies of our group using this approach are Gallart et al. (1996b,c, 1999b); Aparicio et al. (1997a,b); MartínezDelgado et al (1999). Hurley-Keller et al. (1998) use a similar technique. A conceptually analogous method was introduced by Bertelli et al. (1992) (see also Vallenari et al. 1996). A binning procedure is also proposed by Dolphin 
(1997). An early simplified approach, using only luminosity functions was used by the group of Bologna (e.g. Tosi et al. 1991).

The former discussion deals with the method of comparison of CMDs itself, but nothing has been said about the SFH search process. In many of the works that study real data, a set of model CMDs is computed for several different input SFHs among which the best solution is searched (Tolstoy 1996; Gallart et al. 1996a,b; Aparicio et al. 1997a; Hurley-Keller et al. 1998). This approach is a way to overcome the difficulty imposed by the many degrees of freedom of the problem but introduces an intrinsic limitation: the best solution may not be among the checked SFHs. A way to compensate this limitation is checking many models, selecting all those producing an acceptable matching of the observational CMD and taking the average of them as the solution.

However, methods that do not require explicit computation of the model CMDs are preferable. They have the main formal advantage that they require very few or no initial assumptions about the SFH. Examples of them applied to the analysis of real data are Dohm-Plamer et al. (1997); Aparicio et al. (1997b); Mighell (1997); Gallart et al. (1999b) and Hernández et al. (1999b). Dolphin (1997) shows a consistency test using only synthetic data.

In our recent applications (Aparicio et al. 1997b; Gallart et al. 1999b; Gallart et al., this book), observational effects are simulated in the way described in Sec. 3.2, and $Z(t), \phi(m)$ and $\beta(f, q)$ are tested together with $\psi(t)$. Only a model CMD with constant $\psi(t)$ for the whole time interval is required for each combination of $Z(t), \phi(m)$ and $\beta(f, q)$. The fact that the model CMDs keep a record of the age of every star is used. The distribution of stars in the boxes defined in the model CMDs are computed separately for narrow age intervals. For formal purposes only, we will call $\Delta_{i}(t)$ the step functions defining these time intervals: $\Delta_{i}(t)=1$ for $t$ inside the $i$-th time interval; $\Delta_{i}(t)=0$ otherwise.

The distribution of stars that would correspond to any $\psi(t)$ can be calculated as a linear combination of the distributions for every age interval:

$$
N_{j}^{m}=A \sum_{i} \alpha_{i} N_{j i}^{m}
$$

where the subscript indices $i$ and $j$ refer to the narrow age intervals and to the bidimensional bins defined in the CMD respectively, and the superscript index $m$ stands for the distribution in the model CMD. It is this global distribution which is compared with the results for the observational CMD through

$$
\chi^{2}=\sum_{j} \frac{\left(N_{j}^{o}-N_{j}^{m}\right)^{2}}{N_{j}^{o}}
$$

where index $o$ refers to the observational CMD. The SFR can be obtained as

$$
\psi(t)=A \sum_{i} \alpha_{i} \psi_{0} \Delta_{i}(t)
$$

where $\psi_{0}$ is the constant SFR of the synthetic CMD we are using. $\chi^{2}$ is a multidimensional function depending on the $n \alpha_{\mathrm{i}}$ coefficients defining $\psi(t)$. Minimizing $\chi^{2}$ gives the best $\psi(t)$ for the set of $Z(t), \phi(m)$ and $\beta(f, q)$ used. 


\section{Application of the Method: Summary of Galaxies with a Quanti- tative Derivation of the Full-Life SFH}

A major goal of the analysis of the SFH of nearby galaxies is to compare the properties and evolutionary scenarios for a wide variety of them. This requires obtaining quantitative SFHs for the full life of as many galaxies as possible. Figure 4 summarizes the results of this kind presently available. It does not include some detailed works in which, nevertheless, the full history of the object has not been covered. Intentionally, the Magellanic Clouds have not been included because they probably deserve specific reviews (see Harris, Zaritsky \& Grebel in this book and Geha et al. 1998). The most comprehensive review of SFHs for galaxies in the Local Group, including many additional qualitative estimates, is by Mateo (1998).

Galaxies in Fig. 4 are sorted by decreasing recent to averaged SFR. A few general conclusions can be drawn from it:

- Clear traces appear in all the galaxies of an important star formation activity in the early epoch, regardless of whether the galaxy is a dIr or a $\mathrm{dSph} / \mathrm{dE}$.

- Dwarfs classified as dIr are characterized by the presence of an enhanced star formation activity for the last few hundred Myr. This is the case even for galaxies like Pegasus dIr or Antlia, that have sometimes been considered as $\mathrm{dIr} / \mathrm{dSph}$ transition objects.

- While $\mathrm{dSph} / \mathrm{dE}$ show no intense present day or very recent star formation activity, the presence of a non-negligible intermediate-age to young star formation activity is a common fact. Mechanisms must be found for how these galaxies retain gas during extended periods of time to produce further generations of stars.

- For the young stars, where time resolution is much better, bursting star formation seems to be present (see NGC 6822 and Pegasus). This fact has also been beautifully illustrated for Sextans A (Dohm-Palmer et al. 1997), GR8 (Dohm-Palmer et al. 1998) and Leo A (Tolstoy et al. 1998).

- Evidence of relatively long periods of star forming activity followed by long quiescent intervals is found in Carina (see also Marconi et al. this book, for the case of Fornax).

- In the cases where a wide field has been covered, gradients in the stellar populations are found in the sense that the external regions of the galaxies lack a recent star formation activity which is present in the central region. This is the case of Antlia and Phoenix and a similar result has been found in WLM by Minniti \& Zjilstra (1996). Note however that a more accurate analysis of the stellar population, including kinematics, is necessary first to establish whether the external population is a halo-like one, in the sense of tracing the very early time of the galaxy's history. 


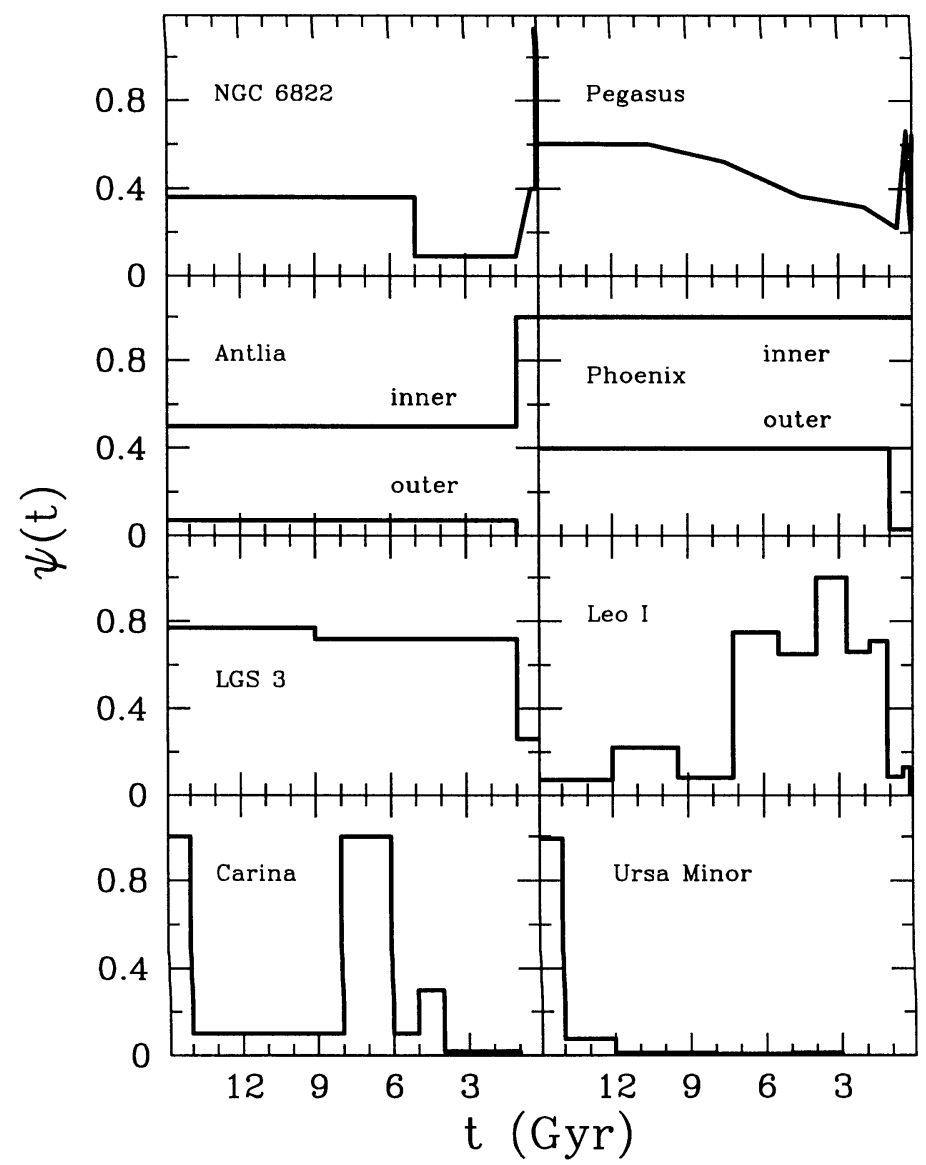

Figure 4. Summary of SFHs of dwarf galaxies, quantitatively determined and extending over the whole life of the system, as presently available in the literature. Normalization $\left(\mathrm{M}_{\odot} \mathrm{yr}^{-1} \mathrm{pc}^{-2}\right.$ that correspond to the unity in the vertical scale), references and comments for each galaxy follow:

NGC 6822: $12 \times 10^{-9}$; Gallart et al. 1996b,c.

Pegasus: $3 \times 10^{-9}$; Aparicio et al. 1997a. See also Gallagher et al. 1998 , specially for the youngest SFH.

Antlia: $6 \times 10^{-10}$; Aparicio et al. $1997 \mathrm{c}$.

Phoenix: $1 \times 10^{-9}$; Martínez-Delgado et al. 1999

LGS 3: $2 \times 10^{-10}$; Aparicio et al. 1997b.

Leo I: $2 \times 10^{-9}$; Gallart et al. $1999 \mathrm{~b}$.

Carina: $5 \times 10^{-9}$; Results from Mighell 1997 and Hurley-Keller et al. 1998, and structural parameters for Carina by Mateo (1998), have been considered.

Ursa Minor: $2.5 \times 10^{-9}$; Martínez-Delgado et al., this book. 


\section{The Next Steps}

Among the several ways in which the research of the SFH can improve, I'd like to emphasize the following three:

\subsection{Deeper}

HST can be used to obtain (i) deeper CMDs of the most nearby objects reaching down to the MS turn-offs of the oldest stars and (ii) CMDs for galaxies in the range of $\sim 5 \mathrm{Mpc}$ of similar depth to those obtained from the ground for galaxies inside the Local Group. Beautiful examples of both kinds start now being available. In particular, Gallart et al. (1999a,b) show for Leo I how HST data can be used to derive reliable solutions for the SFH (including $\psi(t), Z(t)$, $\phi(m)$ and $\beta(f, q))$ for the whole life of galaxies at distances up to several hundred kpc. Hernández et al. (1999b) use HST data of several Milky Way satellites to obtain $\psi(t)$ from a general variational calculus maximum likelihood method. Although these authors introduce simplifications that are probably too strong (no chemical enrichment; no binary stars; Gaussian errors; most observational effects are neglected), the method is a promising one and its full capabilities are still to be developed. Other beautiful examples of detailed $\mathrm{SFH}$, but referred only to the last few hundred Myr are by Dohm-Plamer et al. (1997); Gallagher et al. (1998) and Tolstoy et al. (1998), among others.

On the other side, Lynds et al. (1998) and Schulte-Ladbeck et al. (1998) show how HST CMDs of a galaxy like UGC 6456 , at a distance of $\sim 4.5 \mathrm{Mpc}$ can be used to solve their SFHs with the same level of reliability obtained from the ground for galaxies in the Local Group, such as NGC 6822 (Gallart et al. 1996b,c) or Pegasus (Aparicio et al. 1997a). This is of great importance because it shows the feasibility of obtaining good estimates of the SFH of a large number of galaxies in the vicinity of the Local Group, increasing the knowledge of the properties of galaxies in regions of different densities.

\subsection{Wider Field}

The new generation of wide field cameras for ground-based telescopes can be exploited to study gradients and differentiated disk-like and halo-like structures of the most nearby galaxies. The analysis of Fornax by Stetson et al. (1998) and Phoenix by Martínez-Delgado et al. (1999) are first examples in this direction. Kinematic studies carried on with $10 \mathrm{~m}$ class telescopes should provide fundamental complementary information on the formation and evolutionary history of these systems.

\subsection{Physical Scenarios for the Evolution of Galaxies}

From HST data, the SFR $\psi(t)$ and an estimate of the chemical enrichment law $Z(t)$ can be reliably drawn for the full life of the very nearby galaxies. Both functions can be used together to provide clues on the physical evolution of the galaxies in terms of their interaction with the intergalactic medium.

The "real" or "true" yield $y$ is defined as the mass of newly-formed heavy elements that a generation of stars ejects into the interstellar medium per unit mass locked into stellar remnants or long-lived stars $\left(y=M_{Z} / M_{\star}\right)$. If $y$ is 
known, the metallicity evolution of the interstellar medium can be obtained through (see Peimbert et al. 1994):

$$
\frac{d Z}{d t}=\frac{y s \psi-Z f_{\mathrm{I}}}{M_{\mathrm{g}}},
$$

where $s$ is the fraction of mass that remains locked into stellar remnants and long-lived stars in each generation of stars (instantaneous recycling is assumed), $f_{\mathrm{I}}$ is the infall rate and $M_{\mathrm{g}}$ is the gas mass. $Z, \psi, f_{\mathrm{I}}$ and $M_{\mathrm{g}}$ are, in general functions of time. The gas mass can be obtained as

$$
\frac{d M_{\mathrm{g}}}{d t}=-s \psi+f_{\mathrm{I}}-f_{\mathrm{O}}
$$

with $f_{\mathrm{O}}$ being a function of time accounting for the out-flow rate.

In summary, knowing $\psi(t)$ and $Z(t)$, even if they are just crude estimates, and solving the former equations, limits can be put to $f_{\mathrm{I}}$ and $f_{\mathrm{O}}$ and hence to the physical scenario of galaxy formation and evolution through interaction with the intergalactic medium. The necessary information to face these studies start now being available and is of the kind provided by Gallart et al. (1999b) for Leo I (see also Aparicio et al. 1997b).

\section{Final Considerations: Old and New Preconceptions and Miscon- ceptions about the SFHs of Dwarf Galaxies}

To finish, I want to summarize a few topics that I consider to be or to have been ill-established preconceptions or misconceptions in relation to the SFH of dwarf galaxies. They are not necessarily true or false. They are only concepts that I see or have seen repeated here and there with no solid justification. Some of them seem to be overcome by now. Others have been discussed throughout this paper. Except for items 1 and 4 that are briefly developed in Sec. 6.1 and 6.2, I will only enunciate them with no further comments.

1. Synthetic CMDs are a really very new thing (see Sec. 6.1).

2. Age-metallicity degeneracy largely prevents the possibility of obtaining information about the SFH from the CMD.

3. Stellar evolution models are not good enough for deriving the SFH from synthetic CMDs.

4. Blue plumes in dSph galaxies are certainly blue stragglers (see Sec. 6.2).

5. dIr galaxies have only young stars.

6. dSph galaxies have only old stars.

7. Cosmology is important. The Local Group is not. 


\subsection{Concise Prehistory of the Synthetic CMD}

The following items show how old the idea of the synthetic CMD is.

- The population concept of Baade (e.g. Baade 1944). In the opinion of Sidney van den Bergh (this meeting), this was probably the first step towards the synthetic CMD analysis.

- Maeder (1974) is to my knowledge the first to have made and used a synthetic CMD with a computational monte-carlo technique similar to the one now in use.

- Schild \& Maeder (1983) were the first in my knowledge to compute montecarlo synthetic CMDs applied to the study of the stellar populations in galaxies.

- The ZVAR era. ZVAR, by Bertelli and other members of the Padova group, is probably the code more extensively used for the purpose. Chiosi et al. (1988), Aparicio et al. (1990) and Bertelli et al. (1992) are representative examples of early use of ZVAR. The last is the first example of derivation of the full SFH of a nearby galaxy (the LMC) with synthetic CMDs. It uses the two-dimensional distribution of stars in the CMD and means an important step forward in the accuracy with which the SFH of the LMC was known.

- The Bologna contribution marked a step forward in the systematic study of the recent SFH of galaxies with monte-carlo synthetic CMDs. Tosi et al. (1991) was the first work of this group on this topic.

- And so on...

\subsection{Blue Stragglers?}

About this topic, I just reproduce a sentence by Kenneth Mighell in the coffeeline: "after time and work, every blue-straggler sequence in a dSph has become a complex, intermediate-age population".

Acknowledgements. The expenses of my participation in this conference have been covered by the organization of the meeting, the IAC (grant P3-94) and the DGICyT (grant PB94-0433). I am also very grateful to the persons involved in the organization of the meeting for their permanent and very useful help to solve every kind of small and big problems. I am grateful to Drs. Buonanno, Pritchet and Stetson for allowing me reproducing their CMDs in Figs. 1 and 2.

\section{References}

Aparicio, A., Bertelli, G., Chiosi, C., García-Pelayo, J.M. 1990, A\&A, 240, 262 Aparicio, A., Gallart, C. 1994, in: The Local Group: comparative and global properties, ESO Conference and Workshop Proceedings No. 51. (eds.) A. Layden, R.C. Smith \& J. Storm, p. 115.

Aparicio, A., Gallart, C. 1995, AJ, 110, 2105 
Aparicio, A., Gallart, C., Bertelli, G. 1997a, AJ, 114, 669

Aparicio, A., Gallart, C., Bertelli, G. 1997b, AJ, 114, 680

Aparicio, A., Dalcanton, J.J., Gallart, C., Martínez-Delgado, D. 1997c, AJ, 114, 1447

Aparicio, A., Gallart, C., Chiosi, C., Bertelli, G. 1996, ApJ, 469, L97

Baade, W. 1944, ApJ, 100, 137

Bertelli, G., Mateo, M., Chiosi, C., Bressan, A. 1992, ApJ, 388, 400

Buonanno, R., Corsi, C.E., Fusi Pecci, et al. 1985, A\&A, 152, 65

Chiosi, C., Bertelli, G., Bressan, A. 1992, ARA\&A, 30, 235

Chiosi, C., Bertelli, G., Bressan, A. 1988, A\&A, 196, 84

Da Costa, G.S. 1998, in: Stellar Astrophysics for the Local Group, (eds.) A. Aparicio, A. Herrero \& F. Sánchez, CUP, p. 351

Dohm-Plamer, R.C., Skillman, E.D., Saha, A., et al. 1997, AJ, 114, 2527

Dohm-Plamer, R.C., Skillman, E.D., Gallagher, J., et al. 1998, AJ, 116, 1227

Dolphin, A. 1997, New Astro., 2, 397

Fox, M.F., Pritchet, C.J. 1987, AJ, 93, 1381

Gallagher, J., Tolstoy, E., Dohm-Palmer, R.C., et al. 1998, AJ, 115, 1869

Gallart, C., Aparicio, A., Bertelli, G., Chiosi, C. 1996b, AJ, 112, 1950

Gallart, C., Aparicio, A., Bertelli, G., Chiosi, C. 1996c, AJ, 112, 2596

Gallart, C., Aparicio, A., Vílchez, J.M. 1996a, AJ, 112, 1928

Gallart, C., Freedman, W.L., Aparicio, A., et al. 1999b, AJ, submitted

Gallart, C., Freedman, W., Mateo, M., et al. 1999a, ApJ, in press

Geha, M.C., Holtzman, J.A., Mould, J.R., et al. 1998, AJ, 115, 1045

Hernández, X., Gilmore, G., Valls-Gabaud, D. 1999b, in preparation

Hernández, X., Valls-Gabaud, D., Gilmore, G. 1999a, MNRAS, submitted

Hurley-Keller, D., Mateo, M., Nemec, J. 1998, AJ, 115, 1840

Lynds, R., Tolstoy, E., O’Neil, E.J., Hunter, D. 1998, AJ, 116, 146

Maeder, A. 1974, A\&A, 32, 177

Mighell, K.J. 1997, AJ, 114, 1458

Martínez-Delgado, D., Gallart, C., Aparicio, A. 1999, AJ, submitted

Martínez-Delgado, D., Aparicio, A. 1997, ApJ, 480, L107

Mateo, M. 1998, ARA\&A, 36, 435

Minniti, D., Zijlstra, A.A. 1996, ApJ, 467, L13

Ng, Y.K. 1998, A\&AS, 132, 133

Peimbert, M., Colín, P., Sarmiento, A. 1994, in: Violent Star Formation From 30 Doradus to QSOs, (ed.) G. Tenorio-Tagle, CUP, p. 79

Schild, H., Maeder, A. 1983, A\&A, 127, 238

Schulte-Ladbeck, R.E., Crone, M.M., Hopp, U. 1998, ApJ, 493, L23

Serra-Ricart, M., Aparicio, A., Garrido, L., Gaitan, V. 1996, ApJ, 462, 2221

Stetson, P.B. 1994, PASP, 106, 250

Stetson, P.B., Hesser, J.E., Smecker-Hane, T.A. 1998, PASP, 110, 533 
Tolstoy, E., Gallagher, J.S., Cole, A.A., et al. 1998, AJ, 116, 1244

Tolstoy, E., Saha, A. 1996, ApJ, 462, 672

Tolstoy, E. 1996, ApJ, 462, 684

Tosi, M., Greggio, L., Marconi, G., Focardi, P. 1991, AJ, 102, 951

Vallenari, A., Chiosi, C., Bertelli, G., et al. 1996, A\&A, 309, 367

\section{Discussion}

Terndrup: I'm pleased to see the sophistication of this analysis in which you can whittle down the parameters space of stars which could possibly be in these galaxies to leave the stars which plausibly are there. But are the solutions physical in a chemical evolution sense? Are you planning to investigate this in more detail in the future?

Traap: Deriving the SFH and the metallicity enrichment law from CMDs of galaxies, how can you guarantee the evolutionary consistency on solutions?

Aparicio: In fact, that is one of the steps forward to be done now. See the explanation in section 5.3 which was included after the comments by Terndrup and Traap.

Bellazzini: In comparisons between synthetic and observed CMDs, you explore a large portion of a many dimension parameter space. It would be very interesting to look in detail at the shape of the minimum to check how many different solutions are effectively found and how different they are.

Aparicio: That's right. The multiplicity of solutions is a problem that needs careful attention. We are working in the sense you suggest.

Van den Bergh: The history of synthetic CMDs starts with Baade (1944) and the two components (populations I and II) model. Later, Morgan (1957) showed that life was more complicated because the central bulge of M31 contains a metal-rich old population, so one needs more components.

Aparicio: The first item of section 6.1 was included after this comment by Van den Bergh. 